\title{
Schematic model for QCD at finite temperature
}

\author{
S. Lerma H., ${ }^{*}$ S. Jesgarz, ${ }^{\dagger}$ P. O. Hess, ${ }^{1, \$}$ O. Civitarese, ${ }^{2, \S}$ and M. Reboiro ${ }^{2, \|}$ \\ ${ }^{1}$ Instituto de Ciencias Nucleares, Universidad Nacional Autónoma de México, Apdo. Postal 70-543, 04510 D.F., México \\ ${ }^{2}$ Departamento de Física, Universidad Nacional de La Plata, c.c. 67 1900, La Plata, Argentina \\ (Received 28 May 2002; revised manuscript received 12 July 2002; published 29 October 2002)
}

\begin{abstract}
The simplest version of a class of toy models for QCD is presented. It is a Lipkin-type model, for the quark-antiquark sector, and, for the gluon sector, gluon pairs with spin zero are treated as elementary bosons. The model restricts to mesons with spin zero and to few baryonic states. The corresponding energy spectrum is discussed. We show that ground state correlations are essential to describe physical properties of the spectrum at low energies. Quantum phase transitions are described in an effective manner, by using coherent states. The appearance of a Goldstone boson for large values of the interaction strength is discussed, as related to a collective state. The formalism is extended to consider finite temperatures. The partition function is calculated, in an approximate way, showing the convenience of the use of coherent states. The energy density, heat capacity, and transitions from the hadronic phase to the quark-gluon plasma are calculated.
\end{abstract}

DOI: 10.1103/PhysRevC.66.045207 PACS number(s): 24.85.+p, 12.38. $-\mathrm{t}, 12.39 .-\mathrm{x}, 21.65 .+\mathrm{f}$

\section{INTRODUCTION}

Schematic models have been very important in order to understand basic concepts in, e.g., nuclear physics (pairing, quadrupole interaction, quantum phase transition from spherical to deformed nuclei, etc.). In particular, Lipkin-type models [1] were important in this context. For example, in Ref. [2] the quantum phase transition to a pion condensate was investigated and in Ref. [3] a model was presented which describes the coupling of fermion and boson degrees of freedom. In the description of the $\beta \beta$ decay, simple models $[4,5]$ helped us to understand the applicability of different many-body methods by comparing approximations to the exact solution of the schematic model. In Ref. [6] many considerations were dedicated to the Lipkin type models in order to illustrate boson mapping techniques. In summary, with the help of schematic models not only can a physical insight be achieved but also different many-body techniques can be tested; both features are important for theories where an exact or approximate solution cannot be achieved easily, at all. We are convinced that a similar model for QCD can be very useful in order to understand the spectrum of QCD at low energy, its structure with respect to quantum phase transitions and the transition to the quark-gluon-plasma (QGP), without the need to combine models which are valid at low energy with others which are valid at high energy.

QCD is considered to be the theory of the strong interactions. It is well understood at high energies. At low energies, the QCD coupling constant becomes too large to apply perturbation theory. Lattice gauge calculations [7] may describe the nonperturbative QCD regime, instead. Yet, problems like finite size effects [8] and fermion doubling still persist. Although some advances have been made, only the lowest

\footnotetext{
*Email address: alerma@nuclecu.unam.mx

†Email address: jesgarz@nuclecu.unam.mx

*Email address: hess@nuclecu.unam.mx

$\S$ Email address: civitare@venus.fisica.unlp.edu.ar

"Email address: reboiro@venus.fisica.unlp.edu.ar
}

states for a given spin and parity can be calculated. The observed sequence of levels cannot be explained by lattice gauge calculations, and alternative methods have to be developed to explain the ordering, as, e.g., done in Ref. [9]. Many effective models have achieved some success in describing the low energy regime of QCD $[10,11]$. These models have in common that only quarks and antiquarks are taken into account in the fermionic sector, while effective gluon potentials or states with a fixed number of gluons are considered. In the real world hadrons are built by quarks, antiquarks, and gluons [12]. The interactions between these degrees of freedom, in consequence, may play an essential role in order to understand QCD at low energies.

Concerning QCD effects at finite temperature, i.e., the investigation of the quark-gluon plasma (QGP) [13], there exists an intense effort, mainly focused on computational aspects of the problem [14].

In this paper, we propose a toy model for QCD which: (i) is amenable for an analytical treatment, except for a numerical matrix diagonalization, (ii) may describe the meson spectrum for flavor $(0,0)$-spin 0 and partly the baryon spectrum, (iii) it is able to describe quantum phase transitions at $T$ $=0$, as a function of coupling parameters, (iv) can describe some characteristics of the transition from the hadron gas to the QGP continously from $T=0$ to large $T$, and (v) can be used to test microscopic many-body techniques, intended to describe realistic scenarios of QCD which cannot be accessed by other methods. (Because the system is of finite number of degrees of freedom, in the strong sense no phase transition can appear. However, it will be smeared out and the notion of phase transition is used in the same way as in nuclear physics, where sharp localized changes in the energy or its derivatives are denoted as a phase transition.)

The model is meant to mock up the basis features of nonperturbative QCD, in a similar way as some schematic models do in the nuclear many-body problem $[1,2]$. We should stress that our toy model does not result from a field theory, rather it represents the interactions between effective degrees of freedom, instead. The basic ideas, procedures, and methods can be discussed already for the simplest versions of the 


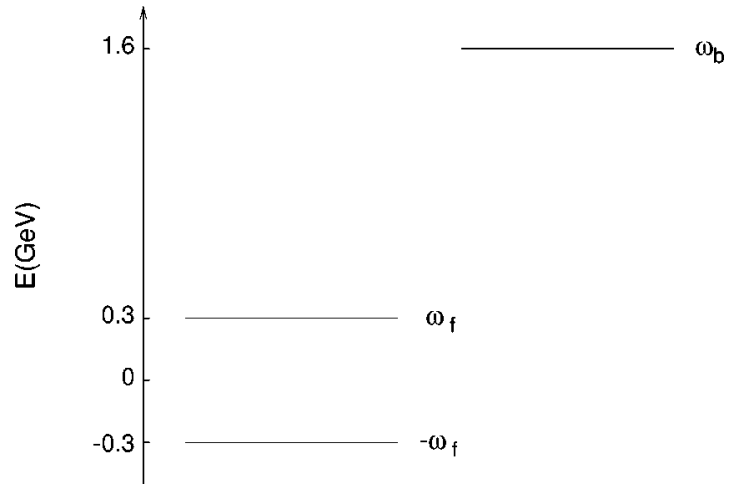

FIG. 1. Schematic representation of the model space. The fermion levels are indicated by their energies $\pm \omega_{f}$. The gluon pairs are represented by the level at the energy $\omega_{b}$.

toy model in a very transparent way. At a certain point in our discussion we shall indicate the structure of an extended version of the model, which can be treated analytically. The model will be able to describe explicitly quantum phase transitions at zero temperature $(T=0)$ and the phase transition to the QGP. It is, for the moment, the only model which can do it continuously from $T=0$ to high $T$. Of course, we have to pay a price for that, i.e., the loss of the ability to describe QCD in all its details.

The paper is organized as follows. In Sec. II the model is introduced, the energy spectrum is calculated, and its structure is discussed in terms of the elementary degrees of freedom. Coherent states are introduced to determine the occurrence of quantum phase transition, induced by variations of the strength of the interactions. In Sec. III we discuss finite temperature effects, by introducing temperature and by calculating the grand canonical partition function. There, a method will be presented which can be applied to arbitrary realistic Hamiltonians and is therefore of use in more general and realistic theories. Conclusions are drawn in Sec. IV.

\section{TOY MODEL: ZERO TEMPERATURE CASE}

The fermion sector of the theory is described by the action of the operators which create (annihilate) quarks, with effective masses $\omega_{f}$. Schematically, it corresponds to the situation represented in Fig. 1, where two levels, with energy $\pm \omega_{f}$ and degeneracy $2 \Omega$, are represented as valence space [15]. The degeneracy of each level is given by the product of the number of colors $\left(n_{c}\right)$, spin $\left(n_{S}\right)$, flavors $\left(n_{f l}\right)$, and all other possible degrees of freedom $\left(n_{\text {col }}\right)$, like orbital quantum numbers, etc. For temperature $T=0$ and no interaction the lower level is filled by fermions. The creation (annihilation) operators of these fermions are $\boldsymbol{c}_{\alpha(1,0) f \sigma i}^{\dagger}\left(\boldsymbol{c}^{\alpha(1,0) f \sigma i}\right)$, in coand contravariant notation for the indices. The symbol $(1,0) f$ refers to the flavor part, where $(1,0)$ is the SU(3)-flavor notation and $f$ is a shorthand notation for the hypercharge $Y$, the isospin $T$, and its third component $T_{z}$. The index $\sigma$ represents the two spin components $\pm \frac{1}{2}$, the index $i=1$ or 2 stands for the upper or lower level, and the index $\alpha$ represents all remaining degrees of freedom, which are at least 3 if the color degree of freedom is considered. Lowering and raising the indices of the operators introduces a phase, which depends on the convention used [16], and a change of the indices to their conjugate values, i.e., the quantum numbers $(1,0) Y T T_{z} \sigma$ change to $(0,1)-Y T-T_{z}-\sigma$.

The operators, defined above, contain the most important degrees of freedom of QCD, i.e., color, spin, and flavor which are the same for all energies. This is mainly due to the difference in the spatial properties, while color, spin, and flavor are still the same. The quarks and antiquarks are constituent particles at low energy and have little in common (except for the some quantum numbers) with the ones at high energy. One lesson to learn will be that a model which contains these basic degrees of freedom and takes into account the dynamic coupling with gluons can describe the main characteristics of QCD at low energy.

The quark and antiquark creation and annihilation operators are given in terms of the operators $c$ and $c^{\dagger}$,

$$
\begin{gathered}
\boldsymbol{a}_{\alpha f \sigma}^{\dagger}=\boldsymbol{c}_{\alpha f \sigma 1}^{\dagger}, \quad \boldsymbol{d}_{\alpha f \sigma}=\boldsymbol{c}_{\alpha f \sigma 2}^{\dagger}, \\
\boldsymbol{a}^{\alpha f \sigma}=\boldsymbol{c}^{\alpha f \sigma 1}, \quad \boldsymbol{d}^{\dagger \alpha f \sigma}=\boldsymbol{c}^{\alpha f \sigma 2},
\end{gathered}
$$

which corresponds to the Dirac picture of particles and antiparticles: quarks are described by fermions in the upper level and antiquarks by holes in the lower level.

The gluon sector of the model space is described by bosons which represent pair of gluons coupled to spin zero. The energy of a boson state is fixed at the value $\omega_{b}$ and the state is created (annihilated) by the action of a boson creation (annihilation) operator $\boldsymbol{b}$ on the vacuum.

The quark-antiquark pairs of the model are given by

$$
\begin{gathered}
\boldsymbol{C}_{f_{1} \sigma_{1} 1}^{f_{2} \sigma_{2} 2}=\boldsymbol{B}_{f_{1} \sigma_{1}}^{\dagger f_{2} \sigma_{2}}=\sum_{\alpha} \boldsymbol{c}_{\alpha f_{1} \sigma_{1} 1}^{\dagger} \boldsymbol{c}^{\alpha f_{2} \sigma_{2} 2}=\sum_{\alpha} \boldsymbol{a}_{\alpha f_{1} \sigma_{1}}^{\dagger} \boldsymbol{d}^{\dagger \alpha f_{2} \sigma_{2}}, \\
\boldsymbol{C}_{f_{1} \sigma_{1} 2}^{f_{2} \sigma_{2} 1}=\boldsymbol{B}_{f_{1} \sigma_{1}}^{f_{2} \sigma_{2}}=\sum_{\alpha} \boldsymbol{c}_{\alpha f_{1} \sigma_{1} 2}^{\dagger} \boldsymbol{c}^{\alpha f_{2} \sigma_{2} 1}=\sum_{\alpha} \boldsymbol{d}_{\alpha f_{1} \sigma_{1}} \boldsymbol{a}^{\alpha f_{2} \sigma_{2}}, \\
\boldsymbol{C}_{f_{1} \sigma_{1} 1}^{f_{2} \sigma_{2} 1}=\sum_{\alpha} \boldsymbol{c}_{\alpha f_{1} \sigma_{1} 1}^{\dagger} \boldsymbol{c}^{\alpha f_{2} \sigma_{2} 1}=\sum_{\alpha} \boldsymbol{a}_{\alpha f_{1} \sigma_{1}}^{\dagger} \boldsymbol{a}^{\alpha f_{2} \sigma_{2}} \\
\boldsymbol{C}_{f_{1} \sigma_{1} 2}^{f_{2} \sigma_{2} 2}=\sum_{\alpha} \boldsymbol{c}_{\alpha f_{1} \sigma_{1} 2}^{\dagger} \boldsymbol{c}^{\alpha f_{2} \sigma_{2} 2}=\sum_{\alpha} \boldsymbol{d}_{\alpha f_{1} \sigma_{1}} \boldsymbol{d}^{\dagger \alpha f_{2} \sigma_{2}} .
\end{gathered}
$$

The first two equations describe the creation and annihilation of quark-antiquark pairs. The pairs can be coupled to definite flavor $(\lambda, \lambda)=(0,0)$ or $(1,1)$ and $\operatorname{spin} S=0$ or 1 . We shall write, in this coupling scheme, $\boldsymbol{B}_{(\lambda, \lambda) f, S M}^{\dagger}$, where $f$ is the flavor, $S$ is the spin, and $M$ is the spin projection. The operators $\boldsymbol{B}_{(\lambda, \lambda) f, S M}$ annihilate the vacuum $|0\rangle$, which is the configuration where the lower state is completely filled and the upper one is empty. The operators in Eq. (2) form a U(12) algebra [17]. To simplify the discussion, we shall restrict it to a subalgebra given by the pair operators coupled to flavor singlet $[(0,0)$ in the $\mathrm{SU}(3)$ notation [18]],

$$
\begin{aligned}
& \boldsymbol{S}_{+}=\sqrt{6} \boldsymbol{B}_{(0,0) 0,00}^{\dagger}, \\
& \boldsymbol{S}_{-}=\sqrt{6} \boldsymbol{B}_{(0,0) 0,00},
\end{aligned}
$$




$$
\boldsymbol{S}_{0}=\boldsymbol{n}_{f}-\Omega,
$$

with $n_{f}=\left(n_{q}+n_{q}\right) / 2$, where $n_{q}$ is the number operator for quarks and $n_{q}^{-}$the number operator for the antiquarks. They form a $\mathrm{SU}(2)$ algebra.

In a subsequent publication [17] the more general model will be discussed. In this contribution we center around this simplest version in order to illustrate the basic concepts, though as we will see, already here several conclusions about the structure of states at low energy (low lying collective states and mixing of particle numbers) can be drawn and also for the transition to the QGP.

The model is equivalent to the Lipkin model [1], familiar in nuclear physics, with the difference that the operators are given here by the combination of quark-antiquark pairs. The addition of an extra boson level was also discussed in Ref. [2] and it is related to pion effects in nuclei.

As Hamiltonians we shall consider two different types:

$$
\begin{gathered}
\boldsymbol{H}_{I}=2 \omega_{f} \boldsymbol{S}_{0}+\omega_{b} \boldsymbol{n}_{b}+V_{1}\left(\boldsymbol{S}_{+}^{2} \boldsymbol{b}+\boldsymbol{b}^{\dagger} \boldsymbol{S}_{-}^{2}\right), \\
\boldsymbol{H}_{I I}=2 \omega_{f} \boldsymbol{S}_{0}+\omega_{b} \boldsymbol{n}_{b}+V_{1}:\left(\boldsymbol{S}_{+}+\boldsymbol{S}_{-}\right)^{2}:\left(\boldsymbol{b}^{\dagger}+\boldsymbol{b}\right),
\end{gathered}
$$

with $\boldsymbol{S}_{0}=\boldsymbol{n}_{f}-\Omega$ and the double dots indicate normal ordering.

The Hamiltonian $H_{I}$ exhibits a useful symmetry, i.e., it commutes with the operator

$$
\boldsymbol{P}=\frac{\boldsymbol{n}_{f}}{2}+\boldsymbol{n}_{b} .
$$

This version of the Hamiltonian is similar to the one given in Ref. [2], except that in Ref. [2] the SU(2) operators appear linearly while in Eq. (4) they appear quadratically. Because a quark-antiquark pair has negative parity, the operators $\boldsymbol{S}_{+}$ and $\boldsymbol{S}_{-}$should appear quadratically to conserve parity.

The states of the model space, belonging to $\boldsymbol{H}_{I}$, are $\mathrm{SU}(2)$ states with the additional ordering given by the eigenvalues of $\boldsymbol{P}$. The vacuum state is defined via $\boldsymbol{b}|0\rangle=\boldsymbol{S}_{-}|0\rangle=0$. Because the number of fermion pairs $n_{f}$ is limited by $2 \Omega$ the range of $n_{b}$ for a fixed value of $P$ is also limited. Therefore the matrix representation of $\boldsymbol{H}_{I}$ are finite. A large eigenvalue of $\boldsymbol{P}$ implies that the corresponding configuration has many gluons. In Fig. 2 we show the energy of the lowest state for a given strength of the interaction, as a function of the eigenvalue of $\boldsymbol{P}$. For zero interaction, the energy increases monotonically. For large values of $V_{1}$ it appears a minimum with a large value of $P$. This implies that the physical ground state should be a correlated one, that is to say that the physical ground state will, likely, be a state with a large number of gluon pairs. Concerning the dependence upon $V_{1}$, which is the strength of the interaction which couples pairs of gluons with pairs of quark-antiquark pairs, the curves of Fig. 2 show minima, which are different from the perturbative vacuum, for values of $V_{1} \geqslant 0.035 \mathrm{GeV}$. For larger values of the interaction strength $V_{1}$, the lowest state is the one with a large eigenvalue $P$, indicating a gluon dominated vacuum (for the transitional region, quark-antiquark and gluon pairs could appear in the vacuum with comparable weights). In Fig. 3,

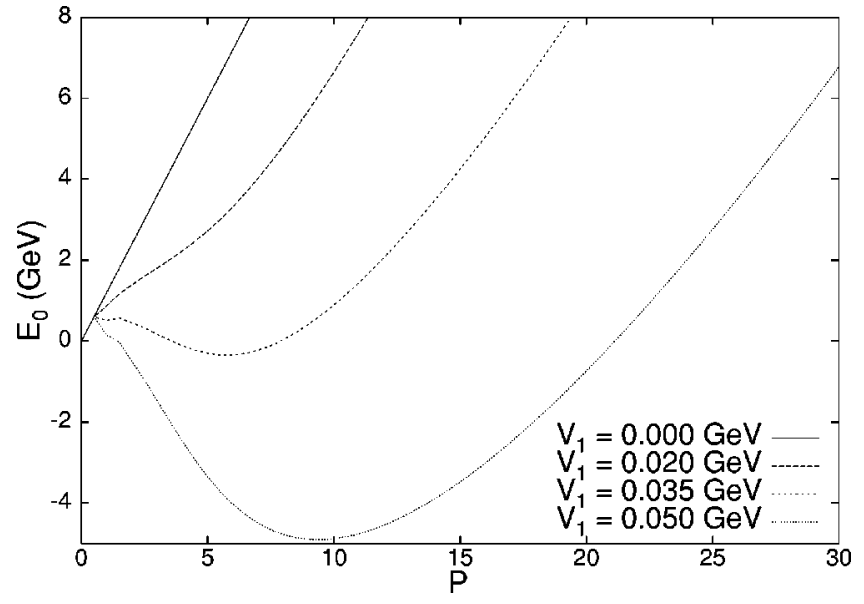

FIG. 2. The energy of the ground state $E_{0}$ in units of $\mathrm{GeV}$, as a function of $P$, for several values of the parameter $V_{1}$. The values correspond to the calculations performed with the Hamiltonian $\boldsymbol{H}_{I}$. Note the occurrence of a nontrivial minima when $V_{1}$ $\geqslant 0.035 \mathrm{GeV}$.

the energy spectrum of $\boldsymbol{H}_{I}$ for positive parity states is displayed. The parameters used in the calculations are: $\Omega=9$ (i.e., $n_{c}=3$ and $n_{f l}=3$ ), $\omega_{f}=\frac{1}{3} \mathrm{GeV}$, and $\omega_{b}=1.6 \mathrm{GeV}$. For $V_{1}=0 \mathrm{GeV}$ we obtain the first state at $\frac{4}{3} \mathrm{GeV}$, corresponding to two quark-antiquark pairs. The next state is the glueball at $1.6 \mathrm{GeV}$. When the interaction is turned on, the energy changes until it reaches a "critical" or "transitional" point at $V_{1}^{c}=0.035 \mathrm{GeV}$. There, a level crossing occurs and the lowest state, for higher values of $V_{1}$, has both quarkantiquark and gluon pairs. Beyond the transitional point, the density of levels increases. This effect is known from nuclear physics, where the transition from a spherical nucleus to a deformed one is accompanied by a significant increase of the density of levels at very low energies [19]. The transition is of second order as can be seen by inspecting Fig. 2. For values of the interaction larger than $V_{1}^{c}$, the ground-state expectation value of the number of quark-antiquark and

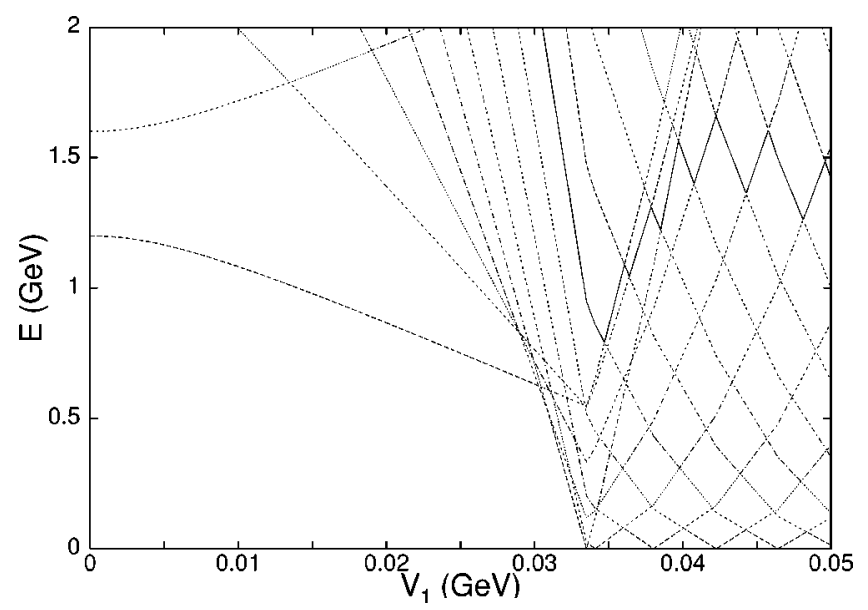

FIG. 3. The spectrum of the model Hamiltonian $\boldsymbol{H}_{I}$, for positive parity states, as a function of the coupling parameter $V_{1}$. Note the crossing of excited states with the perturbative ground state at about $V_{1} \approx 0.035 \mathrm{GeV}$. 

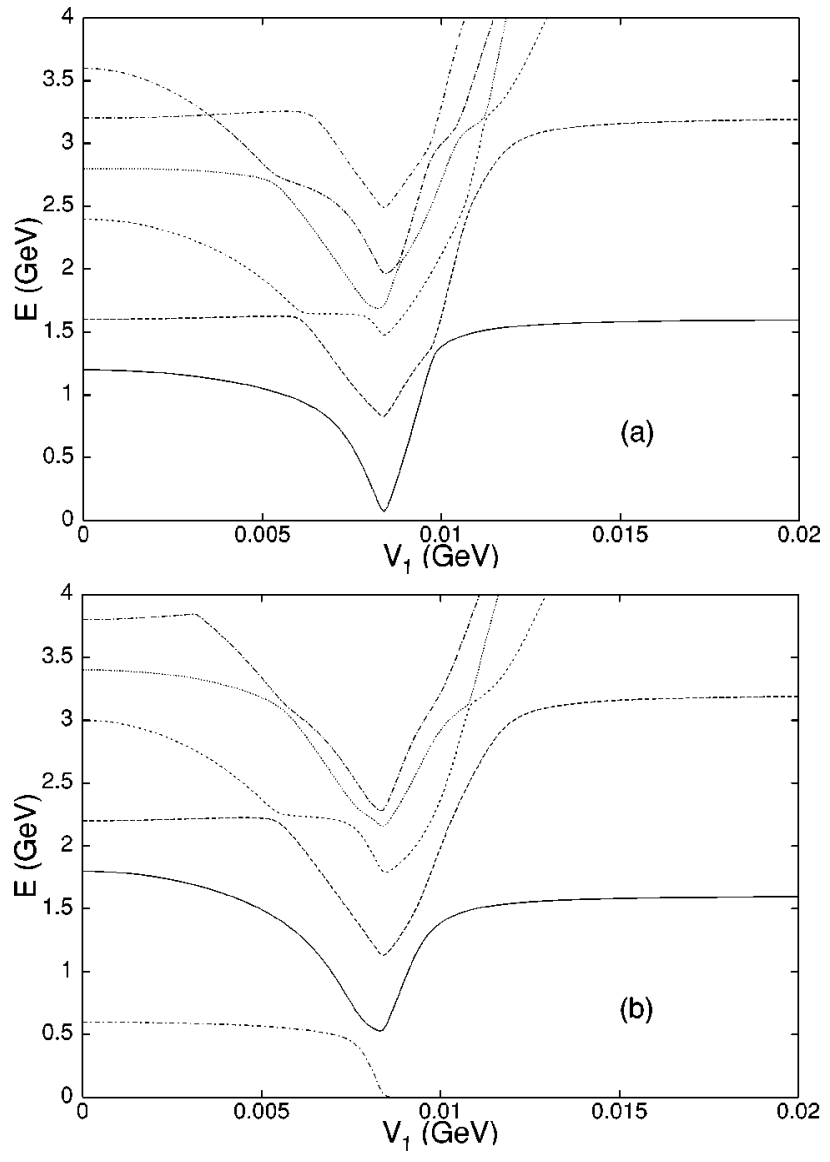

FIG. 4. The energy spectrum of Hamiltonian $\boldsymbol{H}_{I I}$ for positive [case (a)] and negative parity [case (b)] states, as a function of the coupling strength $V_{1}$. The onset of the phase transition takes place for lower values of $V_{1}$, as compared with the results corresponding to the Hamiltonian $\boldsymbol{H}_{I}$ (see Fig. 3).

gluon pairs increases. For large interaction strength the number $n_{f}$ approaches a constant value, reflecting the Pauli principle, i.e., only a certain number of quarks can occupy the higher level. This behavior will be discussed in detail for the case $\boldsymbol{H}_{I I}$. The relatively high density of states at low energy, shown in Fig. 3, may not be very realistic. However, the model predicts the appearance of some states at very low energy. This would correspond to a pionlike structure, which is also indicative of a collective nature.

This problem does not appear in the case of the Hamiltonian $\boldsymbol{H}_{I I}$, which does not commute with $\boldsymbol{P}$. The Hamiltonian $\boldsymbol{H}_{I I}$ has to be diagonalized in the whole space, which is infinite dimensional. The diagonalization can be performed numerically by introducing a variable cutoff in the number of bosons. We have adopted, as a criterium for convergence, the stability of the low-energy sector of the spectrum as a function of the cutoff. The Hamiltonian $\boldsymbol{H}_{I I}$ contains all terms which are required by symmetry, i.e., it includes a term of the form $\boldsymbol{S}_{+}^{2} \boldsymbol{b}$, describing the annihilation of a gluon pair and the creation of two quark-antiquark pairs, and also a term $\boldsymbol{S}_{+}^{2} \boldsymbol{b}^{\dagger}$, describing ground-state correlations. The scattering term $\boldsymbol{S}_{+} \boldsymbol{S}_{-}\left(\boldsymbol{b}^{\dagger}+\boldsymbol{b}\right)$ appears with a factor of 2 because fermion lines can be exchanged. Because of the symmetry in permuting the lines all interactions should have the same (or

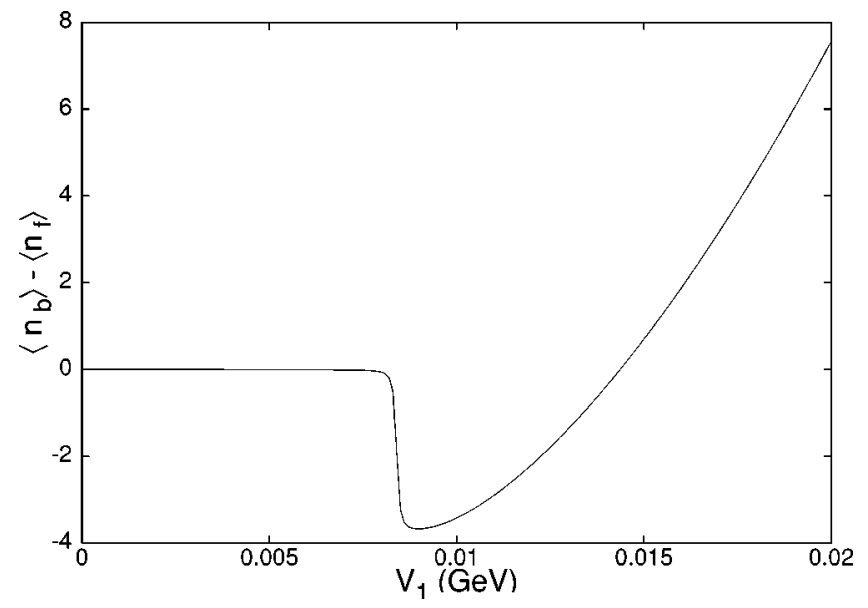

FIG. 5. The difference between the vacuum expectation values of the number of gluon pairs $n_{b}$ and fermion pairs $n_{f}$ is shown, as a function of $V_{1}$, and for the case of the Hamiltonian $\boldsymbol{H}_{I I}$.

at least similar) coupling constant, justifying the use of only one interaction parameter, $V_{1}$. Figure 4 shows the dependence of the energy, of positive [case (a)] and negative [case (b) ] parity states, on the strength $V_{1}$, for the values of $\omega_{f}$ and $\omega_{b}$ given above. The values are referred to the positive-parity ground state. Contrary to the case of the Hamiltonian $\boldsymbol{H}_{I}$, the Hamiltonian $\boldsymbol{H}_{I I}$ does not show a dense spectrum beyond the phase transition point. In the transitional region several avoided crossings occur and the spectrum is richer there, than outside that region. Concerning the behavior of the lowenergy part of the spectrum, it shows, after the transition point, a negative parity state which is degenerate with the positive parity ground state. This state, a Goldstone boson, can be interpreted as a collective, pionlike state whose structure can be understood in the framework of coherent states [20], as it will be discussed later on.

Figure 5 shows for the ground state (vacuum) the difference of the expectation value of the number of gluon and fermion pairs, as a function of the strength $V_{1}$ of the interaction, for the case of the Hamiltonian $\boldsymbol{H}_{I I}$. As it is seen from the figure, the results can be interpreted in terms of equal population of fermion and gluon pairs $\left(V_{1}\right.$ $<0.008 \mathrm{GeV})$, fermionic dominance $\left(0.008<V_{1}\right.$ $<0.015 \mathrm{GeV})$, and gluonic dominance $\left(V_{1}>0.015 \mathrm{GeV}\right)$. Another conclusion concerns the structure of the vacuum state, i.e., it will contain a finite number of quark-antiquark pairs and gluons, whose ratio depends on the strength of the interaction, which in turn has to be adjusted to experiment in the more general model with open flavor and spin [17].

The features of the spectrum, and of the ground-state occupation numbers, can be understood in terms of a quantum phase transition at zero temperature, that is to say, in terms of a change in the correlations induced by the Hamiltonian. A convenient way to represent this effect is to introduce a set of states (coherent states) and to define an order parameter (the ground-state occupation number associated to a given degree of freedom). The calculation of the expectation value of the Hamiltonian, in the basis of coherent states, and its variation with respect to the order parameter yields the possible "phases" of the system, as extremes of the minimization 
procedure. The set of coherent states, which we have adopted in our calculations, is defined by [20]

$$
\begin{gathered}
|z\rangle=\left|z_{f}\right\rangle\left|z_{b}\right\rangle, \\
\left|z_{f}\right\rangle=\frac{1}{\left(1+\left|z_{f}\right|^{2}\right)^{\Omega}} e^{z_{f} \boldsymbol{S}_{+}}|0\rangle_{f}, \\
\left|z_{b}\right\rangle=e^{-\left|z_{b}\right|^{2} / 2} e^{z_{b} b^{\dagger}}|0\rangle_{b},
\end{gathered}
$$

where $|0\rangle_{f}$ and $|0\rangle_{b}$ are the fermion and the boson vacuum and $|0\rangle$ is the product vacuum state $\left(|0\rangle_{f} \otimes|0\rangle_{b}\right)$. The power $\Omega$, of the fermion normalization factor, indicates that we are working in the $\mathrm{SU}(2)$-spin representation which includes, as the lowest state in energy, the fully occupied level at $-\omega_{f}$ (see Fig. 1).

By defining the complex order parameters $z_{f}=\rho_{f} e^{i \phi_{f}}$ and $z_{b}=\rho_{b} e^{i \phi_{b}}$, the expectation value of the Hamiltonian $\boldsymbol{H}_{I I}(4)$ is given by

$$
\begin{aligned}
\left\langle\boldsymbol{H}_{I I}\right\rangle= & -2 \Omega \omega_{f} \frac{\left(1-\rho_{f}^{2}\right)}{\left(1+\rho_{f}^{2}\right)}+\omega_{b} \rho_{b}^{2} \\
& +V_{1}\left(\frac{4 \Omega(2 \Omega-1) \rho_{f}^{2} \cos \left(2 \phi_{f}\right)}{\left(1+\rho_{f}^{2}\right)^{2}}\right. \\
& \left.+\frac{4 \Omega \rho_{f}^{2}\left(2 \Omega+\rho_{f}^{2}\right)}{\left(1+\rho_{f}^{2}\right)^{2}}\right) 2 \rho_{b} \cos \left(\phi_{b}\right),
\end{aligned}
$$

and it can be regarded as a classical potential in the parametric space of the amplitudes $\rho$ and phases $\phi$. It shows for small coupling constants $V_{1}$ a minimum at $\left|z_{f}\right|=\rho_{f}=0$, which represents small departures from a dominant harmonic (quadratic) potential, and a deformed minimum for a sufficiently large value of $V_{1}$ and a given combination of $\phi_{f}$ and $\phi_{b}$. The factor $2 \phi_{f}$, makes the potential invariant under the change $\phi_{f} \longrightarrow \phi_{f}+\pi$. Thus, when the difference in energy of the two minima with respect to the barrier between them is sufficiently large, there exist two degenerate states, one with positive and the other with negative parity, which minimize the expectation value of the Hamiltonian.

The pseudoscalar particle at zero energy cannot be identified with the pion, because the pion belongs to a flavor $(1,1)$ (octet) representation of the flavor group $\mathrm{SU}_{f}(3)$. However, the fact that the model has a Goldstone boson gives some hope that a generalized model with open spin and flavor, as indicated at the beginning, may also exhibit a low lying negative parity state which can be identified with the pion. This conjecture is justified because an equivalent interaction, as in Eq. (4), for flavor $(1,1)$ spin 0 will exhibit a similar behavior with respect to a coherent state which includes pairs with flavor $(1,1)$ spin 0 . One lesson to learn from these results is that the lowest excited energy state will be a combination of quarks-antiquarks and gluons and in the general model the resulting quantum numbers of this state will be the one of the pion.

In our model the basis for the baryonic states is given by

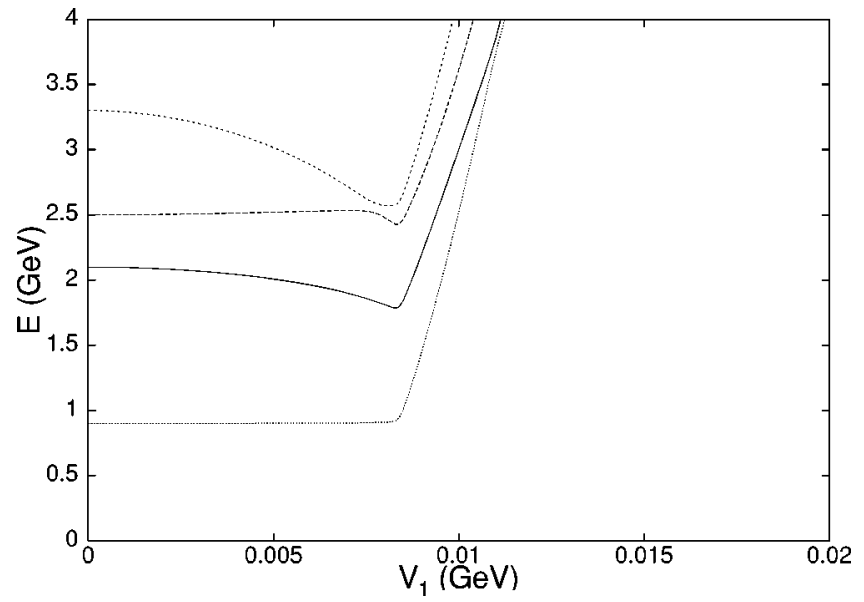

FIG. 6. The spectrum of the lowest baryonic states as a function of the interaction strength. The values of the excitation energies are taken with respect to the ground state of the mesonic sector. The Hamiltonian $\boldsymbol{H}_{I I}$ was used.

$$
\left|q^{3}(q \bar{q})^{n}(\lambda, \mu) f, S M\right\rangle \sim\left(\boldsymbol{S}_{+}\right)^{n}\left|q^{3},(\lambda \mu) f, S M\right\rangle ;
$$

the index $(\lambda, \mu) f$ refers to the flavor, which is $(1,1)$ for the octet, etc., and $q^{3}$ indicates that the state to the right, on which the operator $\boldsymbol{S}_{+}$acts, is a pure three-quark state. The three-quarks state satisfies [11]

$$
\boldsymbol{S}_{-}\left|q^{3},(\lambda \mu) f, S M\right\rangle=0 .
$$

That $\boldsymbol{S}_{-}$annihilates the three-quark state holds because the quark-antiquark pair operator contains an antiquark annihilation operator which anticommutes with the quark creation operators of the state on the right and annihilates finally the correlated vacuum. Note that the Hamiltonian $\boldsymbol{H}_{I I}$ (and the same is true for $\boldsymbol{H}_{I}$ ) does not distinguish between different flavors, and therefore the flavor- $(1,1)$ and flavor- $(3,0)$ baryons are degenerate. Part of the degeneracy can be removed, by introducing terms depending on the hypercharge and the isospin. In order to remove completely the degeneracy between the $(1,1)$ and the $(3,0)$ flavor configurations one has to include, of course, flavor-depending interactions, as it will be done in a more general formulation of the present toy model [17].

The problem for the baryons is completely analogous. Due to the fact that three quarks minimally occupy the higher level of the fermion model space, the effective degeneracy, i.e., the number of configurations available to excite quarks from the lower level, is $2 \Omega-3$ (since the total number of available states is $2 \Omega$ ). The factor 3 is a consequence of the Pauli blocking.

In Fig. 6, the spectrum of the lowest baryonic states, as a function of the coupling strength $V_{1}$ and referred to the lowest positive-parity mesonic state, is shown. After the transition point, the energy of the baryonic states increases. To obtain states below $1 \mathrm{GeV}$, one has to reduce the effective quark mass. Since we are interested in the trends exhibited by the spectrum, we shall not fit it to physical masses. The transition point is slightly shifted to higher values of the coupling constant, due to the lower value of the degeneracy, 


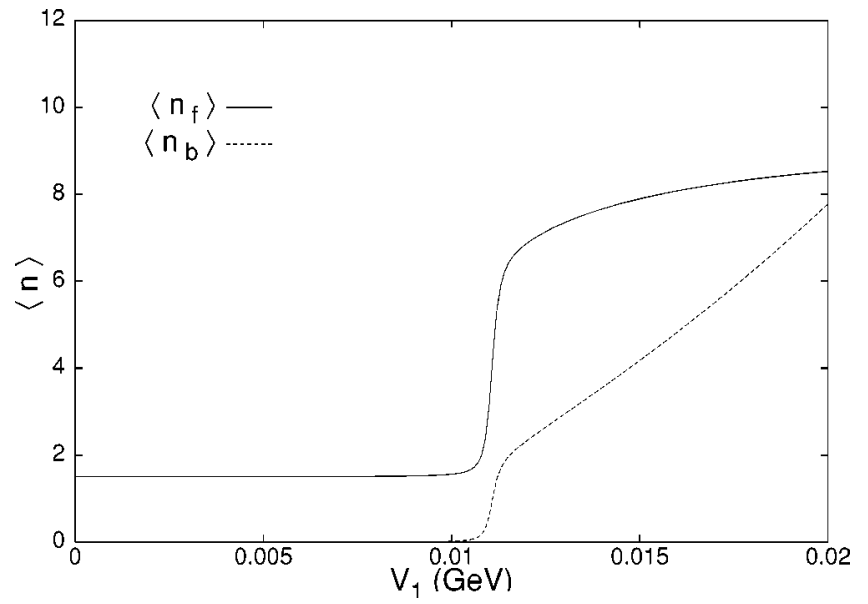

FIG. 7. The content of quark-antiquark $\left(n_{f}\right)$ and gluon $\left(n_{b}\right)$ pairs in the first excited state of the barionic spectrum, as a function of the interaction strength $V_{1}$. The Hamiltonian $\boldsymbol{H}_{I I}$ was used.

otherwise the behavior observed for the baryon spectrum is similar to the meson case. The relatively delayed onset of a transition in the baryon spectra produces a region where the physical vacuum may contain a pair condensate while the baryonic states may still be built as pure three quark objects, i.e., there is a region where the mesons are complicated states in terms of the quarks, antiquarks, and gluons, while the baryons have a simpler structure. Knowing that the baryons have a contribution from the sea quarks and the gluons indicates that probably the mesons are well beyond the point of the quantum phase transition.

In Fig. 7 the content of quark-antiquark and gluon pairs in the first-excited baryonic state is shown. The results show a large contribution from gluon pairs to the baryonic states after the transition point. This indicate that in a more realistic model and for sufficiently large interaction strength, some baryon states, like the proton, may contain a sizable contribution due to sea quarks and gluon pairs, thus shed some light on the microscopic structure of the problem.

After the analysis of the properties of the low lying spectrum of the two Hamiltonians (4), we can proceed to discuss finite temperature effects, which may be relevant for the description of the transition from the hadronic phase to the QGP.

\section{TOY MODEL: FINITE TEMPERATURE CASE}

In this section we shall present the results corresponding to the finite temperature case. We shall show the main steps related to the calculation of the partition function, which has been performed by extending the techniques discussed in Ref. [21].

In the limit $V_{1}=0$, the fermionic sector of the Hamiltonians $\boldsymbol{H}_{I}$ and $\boldsymbol{H}_{I I}$ reduces to the free Lipkin model, which consists of two levels, with energies $\pm \omega_{f}$ and a degeneracy $(2 \Omega)$. Allowed configurations are specified by all possible arrays of particles and holes, in both levels, and their degeneracies. Thus a certain configuration can be specified by listing the number of occupied (empty) states in the upper (lower) level. The operators which create (annihilate) these fermion pairs obey a pseudospin block algebra, for spin $\frac{1}{2}$. There are in total $2 \Omega$ building blocks. If $\nu_{1}$ denotes the number of blocks where both levels are occupied, $\nu_{2}$ is the number of blocks where both levels are empty, and $2 \tau$ is the number of blocks where either one of the levels is occupied, the partition function can be written as [22]

$$
Z(\beta)=\sum_{\tau \nu_{1} \nu_{2}} \frac{(2 \Omega) !}{(2 \tau) ! \nu_{1} ! \nu_{2} !} \sum_{k=0}^{2 \tau} g_{k}^{\tau} I_{\tau-k}
$$

where

$$
I_{J}=\frac{(2 J+1)}{\pi^{2}} \int d^{2} z_{b} \int d^{2} z_{f J} \frac{\left\langle z_{f J}, z_{b}\left|e^{-\beta(\boldsymbol{H}-\mu N)}\right| z_{f J}, z_{b}\right\rangle}{\left(1+\left|z_{f J}\right|^{2}\right)^{2}}
$$

with $J=\tau-k, \beta=1 / T$, and $T$ is the temperature in units of $\mathrm{GeV}$. The states $|z\rangle=|z\rangle_{f} \otimes|z\rangle_{b}$ are the normalized coherent states [20]. The factor $g_{k}^{\tau}$ is the multiplicity of the configuration with pseudo-spin $J=\tau-k$. For each value of $J$ one should define a coherent state $\left|z_{f J}\right\rangle$. The coherent state, used in the previous section (the $T=0$ case), corresponds to the value $J=\Omega$. The chemical potential $\mu$ multiplies the operator $N$, which gives the total number of particles in the lower and upper level. The partition function (10) does not conserve flavor, color, and spin. A multiple projection, to restore these symmetries, can be carried out, in principle, although it is a very involved procedure [13]. We assume that the volume occupied by the QGP is large enough so that a projection is not needed, though we shall restrict to a subvolume for the thermodynamic description.

If interactions are added to the Hamiltonian, the partition function cannot be obtained analytically, in general. If the value of $\Omega$ is not too large, the partition function (10) can be obtained numerically.

We have diagonalized the Hamiltonian $\boldsymbol{H}_{I}$ and obtained a set of eigenvalues for each value of $J$. The parameters of the Hamiltonian were fixed at $V_{1}=0$ and $0.04 \mathrm{GeV}, \Omega=9, \omega_{f}$ $=\frac{1}{3} \mathrm{GeV}$, and $\omega_{b}=1.6 \mathrm{GeV}$. The partition function (10) was calculated for temperature $T<0.5 \mathrm{GeV}$. Since the value of $\Omega$ is not very large, one may ask if, at high temperature, more configurations (i.e., larger values of $\Omega$ ) should be included. We have checked, numerically, this effect upon the partition function. Figure 8 shows the value of Eq. (10), for each value of $J \leqslant \Omega$. As seen from this figure the contributions to the partition function reach a maximum for a certain value of $J$, which is smaller than $\Omega$. This result justifies the approximation with $J \gg 1$. First and second derivatives of the partition function (the internal energy and the heat capacity) are shown in Figs. 9 and 10, respectively. The heat capacity, for $V_{1}=0 \mathrm{GeV}$, shows the Schottky bump [24], typical of a two level system. The shape of the curve remains unchanged when the interaction is switched on in $\boldsymbol{H}_{I}$. The increase of the interaction strength $V_{1}$ produces a sharper peak in the curve, indicating a possible phase transition. The results shown in Figs. 9 and 10 have been obtained with the Hamiltonian $\boldsymbol{H}_{I}$. Although we are not showing it in Figs. 9 and 10, the same thermal behavior of the heat capacity is obtained 


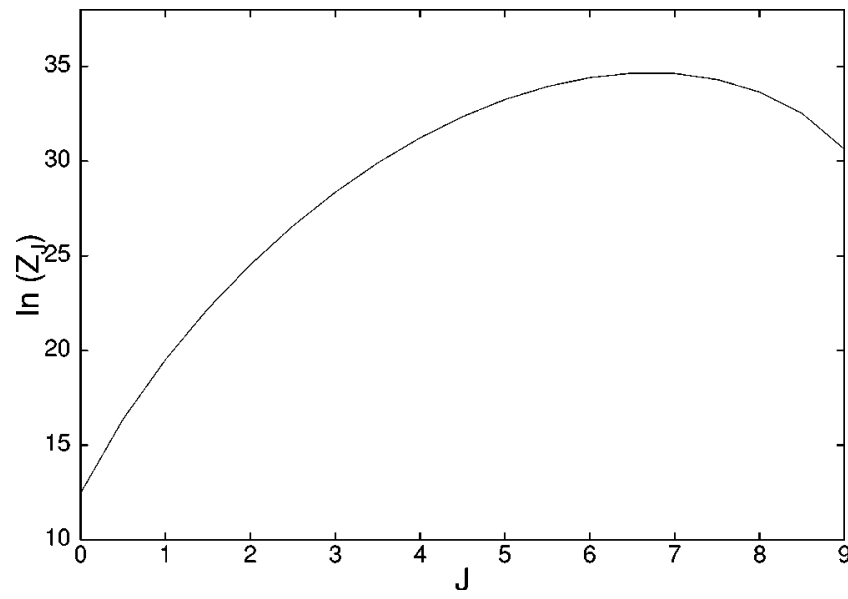

FIG. 8. Contribution to the partition function, Eq. (10), for a fixed value of $J$. The curve shows the logarithm of the results corresponding to the case $\boldsymbol{H}_{I}$, for values of $J \leqslant \Omega=9$, and for $T$ $=0.2 \mathrm{GeV}$, and $V_{1}=0 \mathrm{GeV}$.

with the Hamiltonian $\boldsymbol{H}_{I I}$. However, the values of ( $T$ and $V_{1}$ ), where the onset of the phase transition is produced, are different for the partition functions corresponding to $\boldsymbol{H}_{I}$ and $\boldsymbol{H}_{I I}$. Note that the model predicts a phase transition indicated by the sudden increase of the internal energy at about 0.2 $\mathrm{GeV}$, which may be of first order, as suggested by the results (see Fig. 9).

The above discussed results, which have been obtained by performing a numerical diagonalization, are indeed the exact results of the model. Potentially, they exhibit the desirable thermodynamical features of QCD. We shall take these results as reference values for an approximate calculation. The obvious motivation for such approximate treatment is the generalization to larger values of the model parameters and more general Hamiltonians.

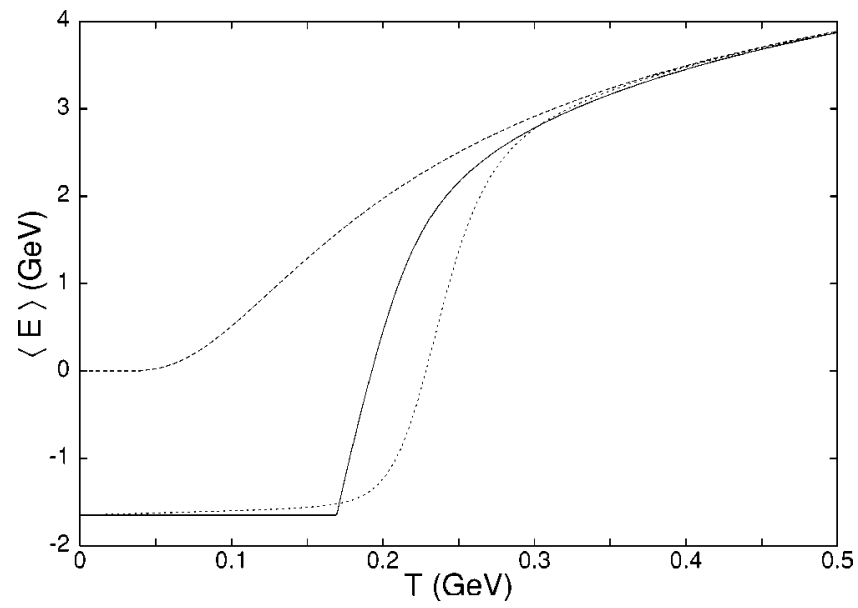

FIG. 9. The temperature dependence of the internal energy, as obtained from the calculations performed with the Hamiltonian $\boldsymbol{H}_{I}$. Dashed lines indicate the exact results corresponding to the unperturbed $\left(V_{1}=0\right)$ case, small-dashed lines show the exact results for $V_{1}=0.04 \mathrm{GeV}$, and the solid line shows the results of the approximations described in Sec. III of the text, see Eq. (29). The chemical potential has the value $\mu=0$.

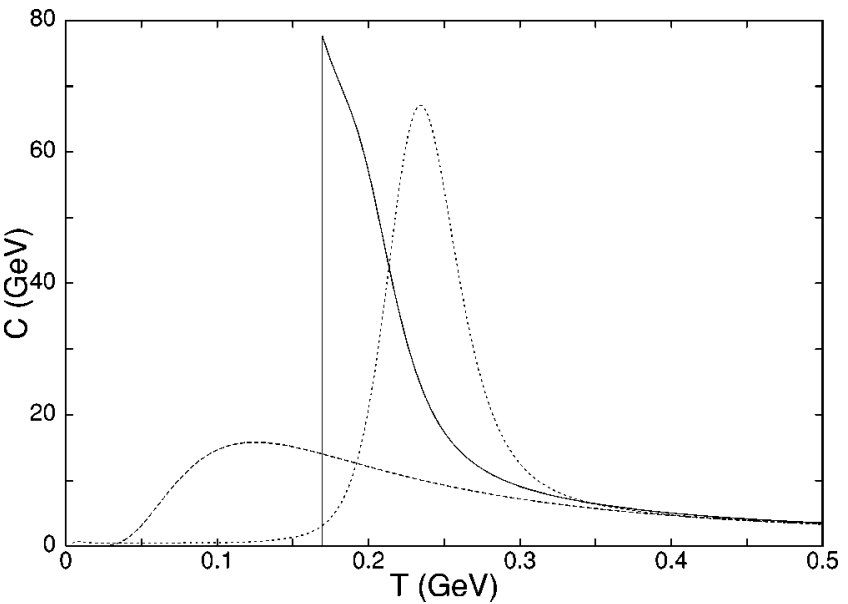

FIG. 10. The specific heat, as a function of temperature, for the cases shown in Fig. 9. Results are shown following the notation given in the captions to Fig. 9.

We shall discuss first the treatment of the fermionic sector, i.e., the integration on the coherent states $\left|z_{\alpha(f J)}\right\rangle$. For large values of $J$, the overlap $\left\langle z_{\alpha} \mid z_{\beta}\right\rangle$ is a decreasing function of $\left|\rho_{\alpha}-\rho_{\beta}\right|$. Also in this limit, it is a strongly oscillating function of the phase difference $\phi_{\alpha}-\phi_{\beta}$. Due to this oscillation we can write, both for $\boldsymbol{H}_{I}$ and $\boldsymbol{H}_{I I}$,

$$
\left\langle z_{\alpha}\left|e^{-\beta \boldsymbol{H}}\right| z_{\beta}\right\rangle \approx\left\langle z_{\alpha}\left|e^{-\beta \boldsymbol{H}}\right| z_{\alpha}\right\rangle\left\langle z_{\alpha} \mid z_{\beta}\right\rangle,
$$

where the overlap in the above equation takes into account the off diagonal behavior for $\alpha \neq \beta$. The expectation value $\left\langle z_{\alpha}\left|e^{-\beta \boldsymbol{H}}\right| z_{\alpha}\right\rangle$ can be expressed as an integral product of the form

$$
\begin{aligned}
& \frac{(2 J+1)}{\pi} \int \frac{d^{2} z_{\alpha}}{\left(1+\left|z_{\alpha}\right|^{2}\right)^{2}}\left\langle z_{\alpha}\left|e^{-\beta \boldsymbol{H}}\right| z_{\alpha}\right\rangle \\
& =\sum_{n=0}^{\infty} \frac{(-\beta)^{n}}{n !} \frac{(2 J+1)^{n}}{\pi^{n}} \int \frac{d^{2} z_{\gamma_{0}} \cdots d^{2} z_{\gamma_{n-1}}}{\Pi_{k=0}^{n-1}\left(1+\left|z_{\gamma_{k}}\right|^{2}\right)^{2}}\left\langle z_{\gamma_{0}}|\boldsymbol{H}| z_{\gamma_{1}}\right\rangle \\
& \quad \times\left\langle z_{\gamma_{1}}|\boldsymbol{H}| z_{\gamma_{2}}\right\rangle \cdots\left\langle z_{\gamma_{n-1}}|\boldsymbol{H}| z_{\gamma_{0}}\right\rangle
\end{aligned}
$$

by inserting $n$ times the unit operator [20]. The Hamiltonian $\boldsymbol{H}$ is expressed into two terms $\boldsymbol{H}_{0}+\boldsymbol{H}^{\prime}$, where $\boldsymbol{H}_{0}$ is the noninteracting part and $\boldsymbol{H}^{\prime}$ contains the interactions. The $k$ th term of the expansion has $k$ matrix elements of $\boldsymbol{H}^{\prime}$ and ( $n$ $-k$ ) factors with $\boldsymbol{H}_{0}$. By performing a rearrangement, assuming also that the main contribution to the integral comes from all $z_{\alpha}$ approximately equal (i.e., it allows renaming $z_{\alpha}$ ), the contribution of the $k$ th partition to the integral is written

$$
\begin{aligned}
\sum_{n=k}^{\infty} & \frac{(-\beta)^{n}}{n !} \frac{(2 J+1)^{2}}{\pi^{2}} \int \frac{d^{2} z_{\alpha} d^{2} z_{\beta}}{\left(1+\left|z_{\alpha}\right|^{2}\right)^{2}\left(1+\left|z_{\beta}\right|^{2}\right)^{2}}\left(\begin{array}{l}
n \\
k
\end{array}\right) \\
& \times\left\langle z_{\alpha}\left|\left(\boldsymbol{H}^{\prime}\right)^{n}\right| z_{\beta}\right\rangle\left\langle z_{\beta}\left|\left(\boldsymbol{H}_{0}\right)^{n-k}\right| z_{\alpha}\right\rangle .
\end{aligned}
$$

This result illustrates the binomial character of the $k$ th partition. By a reexponentiation one can write 


$$
\begin{aligned}
& \frac{(2 J+1)^{2}}{\pi^{2}} \int \frac{d^{2} z_{\alpha} d^{2} z_{\beta}}{\left(1+\left|z_{\alpha}\right|^{2}\right)^{2}\left(1+\left|z_{\beta}\right|^{2}\right)^{2}}\left\langle z_{\alpha}\left|e^{-\beta \boldsymbol{H}_{0}}\right| z_{\beta}\right\rangle \\
& \quad \times\left\langle z_{\beta}\left|e^{-\beta \boldsymbol{H}^{\prime}}\right| z_{\alpha}\right\rangle .
\end{aligned}
$$

One is tempted to say that it corresponds to the assumption that $\boldsymbol{H}_{0}$ and $\boldsymbol{H}^{\prime}$ commute. However, this is not entirely the case because the product appears in an integral and the approximations relies on the assumption of large $J$ and the steep fall off of the overlap. The integral $\int d z_{\alpha}^{2} \int d z_{\beta}^{2}$, can be replaced by a single integral, by using Eq. (12) and by performing the integration on the variable $z_{\beta}$. The validity of this approximation is restricted to large values of $J$, whose validity was shown above. After these approximations (13) reads

$$
\begin{aligned}
& \frac{(2 J+1)^{2}}{\pi^{2}} \int \frac{d^{2} z_{\alpha} d^{2} z_{\beta}}{\left(1+\left|z_{\alpha}\right|^{2}\right)^{2}\left(1+\left|z_{\beta}\right|^{2}\right)^{2}}\left\langle z_{\alpha}\left|e^{-\beta \boldsymbol{H}_{0}}\right| z_{\beta}\right\rangle \\
& \quad \times\left\langle z_{\beta}\right| e^{-\beta \boldsymbol{H}^{\prime}\left|z_{\alpha}\right\rangle} \\
& \quad \approx \frac{(2 J+1)}{\pi} \int \frac{d^{2} z_{\alpha}}{\left(1+\left|z_{\alpha}\right|^{2}\right)^{2}}\left\langle z_{\alpha}\left|e^{-\beta \boldsymbol{H}_{0}}\right| z_{\alpha}\right\rangle\left\langle z_{\alpha}\left|e^{-\beta \boldsymbol{H}^{\prime}}\right| z_{\alpha}\right\rangle .
\end{aligned}
$$

Since $\boldsymbol{H}_{0}=2 \omega_{f} \boldsymbol{S}_{0}$, the matrix element $\left\langle z_{\alpha}\left|e^{-\beta \boldsymbol{H}_{0}}\right| z_{\alpha}\right\rangle$ is readily calculated and the result is

$$
\left\langle z_{\alpha}\left|e^{-\beta 2 \omega_{f} S_{0}}\right| z_{\alpha}\right\rangle=\frac{e^{2 \beta \omega_{f} J}}{\left(1+\left|z_{\alpha}\right|^{2}\right)^{2 J}}\left(1+\left|z_{\alpha}\right|^{2} e^{\left.-2 \beta \omega_{f}\right)^{2 J}} .\right.
$$

As a check on the consistency of these approximations, it is verified that by setting $\boldsymbol{H}^{\prime}=0$ and from the above equation, the integration of Eq. (11) gives a result which is identical to the one of Ref. [22].

Concerning the matrix element of the interaction $\boldsymbol{H}^{\prime}$, we have adopted the following approximation:

$$
\left\langle z_{\alpha}\left|e^{-\beta \boldsymbol{H}^{\prime}}\right| z_{\alpha}\right\rangle \approx e^{-\beta\left\langle<z_{\alpha}\left|\boldsymbol{H}^{\prime}\right| z_{\alpha}\right\rangle},
$$

which is valid when the temperature is high and/or the interaction coupling constant is small. It corresponds to the factorization $\left\langle z_{\alpha}\left|\left(\boldsymbol{H}^{\prime}\right)^{n}\right| z_{\alpha}\right\rangle \approx\left(\left\langle z_{\alpha}\left|\boldsymbol{H}^{\prime}\right| z_{\alpha}\right\rangle\right)^{n}$, a result which can be reproduced, by assuming Eq. (12) and inserting $n$th unit operators between factors $\boldsymbol{H}^{\prime}$. As said before, the applicability of the procedure is limited to relatively small values of the interaction strength $V_{1}$ and relatively large values of the temperature.

Let us now turn the attention to the Bosonic degrees of freedom. The exponent of Eq. (18) is a linear combination of the boson operators $\boldsymbol{b}^{\dagger}$ and $\boldsymbol{b}$. The coefficients are given by the expectation values of $\boldsymbol{S}_{ \pm}^{2}$ or $\boldsymbol{S}_{+} \boldsymbol{S}_{-}$(see the Appendix), which are functions of the complex variables $z_{\alpha}$ and $z_{\alpha}^{*}$. The normalized boson coherent state is given by [20]

$$
\left|z_{b}\right\rangle=e^{-\frac{\left|z_{b}\right|^{2}}{2}} e^{z_{b} b^{\dagger}}|0\rangle .
$$

The volume element of the complex integral is $d^{2} z_{b} / \pi$ (in Ref. [20] the coherent states are not normalized and therefore the volume element has an extra factor $e^{-\left|z_{b}\right|^{2}}$ ). The calculation involves an integral of the type

$$
\frac{1}{\pi} \int d^{2} z_{b}\left\langle z_{b}\left|e^{-\beta \omega_{b} \boldsymbol{n}_{b}+a_{1} \boldsymbol{b}^{\dagger}+a_{2} \boldsymbol{b}}\right| z_{b}\right\rangle .
$$

The coefficients $a_{k}$, which depend on the expectation value of powers of the $\mathrm{SU}(2)$ generators and which are proportional to the interaction strength $V_{1}$, have a common value for the model Hamiltonian $\boldsymbol{H}_{I I}$ but they differ for the case of $\boldsymbol{H}_{I}$.

In order to evaluate the expectation value which appears in Eq. (20), the Baker-Campbell-Hausdorff formula is applied $[20,23]$. Notice that one cannot apply the approximations described for the fermion sector, because the overlaps $\left\langle z_{b} \mid z_{b}^{\prime}\right\rangle$ show a broader dependence on $\rho_{b}-\rho_{b}^{\prime}$ and $\phi_{b}$ $-\phi_{b}^{\prime}$. The result is

$$
e^{-\beta \omega_{b} n_{b}+a_{1} b^{\dagger}+a_{2} b}=e^{\xi_{1} b^{\dagger}} e^{\xi_{2} n_{b}} e^{\xi_{3} b} e^{-K},
$$

with

$$
\begin{aligned}
& K=\xi_{1} \xi_{3}\left[e^{-\xi_{2}}\left(\frac{1}{\xi_{2}^{2}}+\frac{1}{\xi_{2}}\right)-\frac{1}{\xi_{2}^{2}}\right], \\
& \xi_{1}(I)=\frac{2 \beta V_{a}}{2+\beta \omega_{f}}, \quad \xi_{1}(I I)=-\frac{2 \beta V_{c}}{2+\beta \omega_{b}}, \\
& \xi_{2}(I)=-\beta \omega_{b}, \quad \xi_{2}(I I)=-\beta \omega_{b}, \\
& \xi_{3}(I)=-\frac{\beta^{2} \omega_{b} V_{b}}{\left(1-e^{-\beta \omega_{b}}\right)} e^{-\beta \omega_{b}}, \\
& \xi_{3}(I I)=-\frac{\beta^{2} V_{c} \omega_{b}}{\left(1-e^{-\beta \omega_{b}}\right)} e^{-\beta \omega_{b}},
\end{aligned}
$$

and where

$$
\begin{gathered}
V_{a}=V_{1}\left\langle z_{\alpha}\left|\boldsymbol{S}_{-}^{2}\right| z_{\alpha}\right\rangle \\
V_{b}=V_{1}\left\langle z_{\alpha}\left|\boldsymbol{S}_{+}^{2}\right| z_{\alpha}\right\rangle \\
V_{c}=V_{1}\left\langle z_{\alpha}\left|:\left(\boldsymbol{S}_{+}+\boldsymbol{S}_{-}\right)^{2}:\right| z_{\alpha}\right\rangle .
\end{gathered}
$$

The indices $I$ and $I I$ refer to the two different model Hamiltonians.

Because the exponential function which contains the operator $\boldsymbol{b}$ is acting on the coherent state $\left|z_{b}\right\rangle$, the operation is well defined and it gives a factor $e^{\xi_{3} z_{b}}$. The same holds for the exponential function which contains the operator $\boldsymbol{b}^{\dagger}$ and it gives $e^{\xi_{1} z_{b}^{*}}$. The expectation value of the operator which contains $\boldsymbol{n}_{b}$ reads

$$
\left\langle z_{b}\left|e^{\xi_{2} n_{b}}\right| z_{b}\right\rangle=e^{-\left|z_{b}\right|^{2}} e^{\left|z_{b}\right|^{2} e^{\xi_{2}}} .
$$

Finally, the integration over the complex variable $z_{b}$ yields 


$$
\frac{1}{\pi} \int d^{2} z\left\langle z_{b}\left|e^{\xi_{1} b^{\dagger}} e^{\xi_{2} n_{b}} e^{\xi_{3} b}\right| z_{b}\right\rangle=\frac{e^{\xi_{1} \xi_{3} /\left(1-e^{\xi_{2}}\right)}}{1-e^{\xi_{2}}} .
$$

In the following we shall construct the final expression of the partition function, after the above introduced approximations. We shall restrict to the case of $\boldsymbol{H}_{I}$, in order to compare the results of the approximations with the numerical (exact) results. A similar analysis can be performed for the case of
$\boldsymbol{H}_{I I}[17]$. The remaining integration on the complex variable $z_{\alpha}$, of the fermion part, leads to

$$
\begin{aligned}
& \left(\frac{1}{1-e^{-\beta \omega_{b}}}\right)(2 J+1) \int_{0}^{\infty} d x \frac{e^{2 \beta \omega_{f} J}}{(1+x)^{2 J+2}} \\
& \quad \times\left(1+x e^{-2 \beta \omega_{f}}\right)^{2 J} e^{\beta 16 F(\beta) V_{1}^{2} J^{4}\left[x^{2} /(1+x)^{4}\right]},
\end{aligned}
$$

with

$$
F(\beta)=2\left(\frac{1}{\omega_{b}}\right)^{2} \frac{\beta \omega_{b}\left[\left(\beta \omega_{b}\right)^{2}-e^{\left.-\beta \omega_{b}-e^{\beta \omega_{b}}+\beta \omega_{b} e^{\beta \omega_{b}}-\beta \omega_{b}+2\right]}\right.}{\left(2+\beta \omega_{b}\right)\left(e^{\beta \omega_{b}}-1\right)\left(1-e^{-\beta \omega_{b}}\right)},
$$

after substituting $x=\rho^{2} . F(\beta)$ is a smooth function of $\beta$, which approaches the limit $2 \beta / \omega_{b}$ for large $\beta$. The integral, Eq. (26), is a function of the pseudospin $J$ and its argument is the product of exponential functions of positive and negative functions of $J$. That the integral can be represented by the integrand at $J=J_{0}$, where $J_{0}$ is the value that maximizes it, can be easily seen by setting $V_{1}=0$.

Let us call $g(J)$ the ratio between the integral (26) with $V_{1} \neq 0$ and the same integral with $V_{1}=0$, and $J_{0}$ the value of $J$ which maximizes the integral. We can write the Taylor expansion of $g(J)$ as

$$
g(J)=\sum_{n} \frac{1}{n !}\left(\frac{\partial^{n} g}{\partial J^{n}}\right)_{J=J_{0}}\left(J-J_{0}\right)^{n} .
$$

By using the identity

$$
J^{n} X^{2 J}=\frac{1}{2^{n}}\left(\frac{\partial^{n}}{\partial X^{n}} X^{2 J}\right) X^{n},
$$

with $X=e^{\beta \omega_{f}}$, the expansion in terms of $\left(J-J_{0}\right)$ of $g(J)$ transforms into an expansion in the derivatives $\partial / \partial X$. Thus the sum (27) is of the form $\Sigma_{n} D^{n}\left(J_{0}, X\right) Z_{0}$, where $Z_{0}$ is the partition function for the noninteracting case [22] and $D$ is the differential operator $\left[(X / 2)(\partial / \partial X)-J_{0}\right]$. We choose $J_{0}$ such that the $\left[(X / 2)(\partial / \partial X)-J_{0}\right] Z_{0}=0$. The value of $J_{0}$, obtained in this way, is given by

$$
J_{0} \approx \Omega\left(\frac{X^{2} Y}{(X+Y)(1+X Y)}\right)\left(1-\frac{1}{X^{2}}\right),
$$

where $Y=e^{\mu \beta}$ is the fugacity [22]. This expression approaches $J_{0}=\Omega$ for $\beta \rightarrow \infty$ and it gives small values of $J_{0}$ for $\beta \rightarrow 0$. The $g\left(J_{0}\right)$ can be put outside of the sum given in Eq. (10) and the rest can be summed up as in the noninteracting case [22].

After these approximations the partition function can be written as

$$
Z=Z_{f} Z_{b} Z_{\text {int }},
$$

where $Z_{f}$ and $Z_{b}$ are the partition functions for free fermions [22] and free bosons, respectively, i.e.,

$$
\begin{gathered}
Z_{f}=\frac{(X+Y)^{2 \Omega}(1+X Y)^{2 \Omega}}{X^{2 \Omega}}, \\
Z_{b}=\frac{1}{1-e^{-\beta \omega_{b}}},
\end{gathered}
$$

and $Z_{\text {int }}=g\left(J_{0}\right)$ is the contribution due to the interactions. As mentioned above, the value of $g\left(J_{0}\right)$ is given by the ratio of the integral in Eq. (26) with $V_{1}$ different from zero and the same integral with $V_{1}=0$ and $J=J_{0}$. The integral with $V_{1}$ different from zero is calculated numerically, while for $V_{1}$ $=0$ there is an analytical solution given by [without the factor $Z_{b}$ appearing in Eq. (26)]

$$
\frac{e^{2 \beta \epsilon(J+1 / 2)}-e^{-2 \beta \epsilon(J+1 / 2)}}{e^{\beta \epsilon}-e^{-\beta \epsilon}} .
$$

For $\beta \rightarrow 0 Z_{\text {int }} \rightarrow 1$ by construction, and the high temperature limit is automatically obtained. The partition function will be well approximated for large $T$. For small $T$, especially $T=0$, the main contribution to the exact partition function will be $\exp \left(-\beta E_{0}\right)$, where $E_{0}$ is the ground-state energy which can always be determined using coherent states or another variational procedure. For the internal energy it will give a straight line at $E=E_{0}$. We take as the total result the approximation described above until the curve crosses the straight line at $E=E_{0}$.

In what follows we choose the same values of interaction strength as done above when the partition function was calculated numerically.

In Figs. 9 and 10 we show the internal energy and the heat capacity respectively, as a function of the temperature, obtained with the approximate partition function (29). As it can be seen from the curves, the approximation works reasonable well for high temperatures, $T>0.15 \mathrm{GeV}$. At low temperatures the curves, representing the approximated values, devi- 


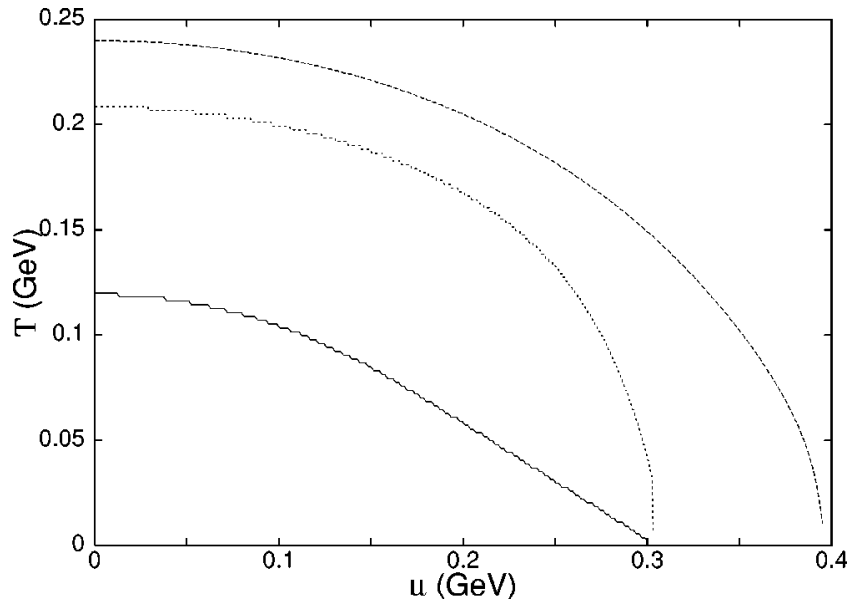

FIG. 11. Temperature as a function of the chemical potential. The solid line indicates the exact results corresponding to the unperturbed $\left(V_{1}=0\right)$ case, the dashed line shows the exact results for $V_{1}=0.04 \mathrm{GeV}$, and the small-dashed line shows the results of the approximations described in Sec. III.

ate significantly from the exact results. It will overshoot the straight line given by $E=E_{0}$ and end at $E=-13 \mathrm{GeV}$ for $T=0$.

The behavior of the curves of Fig. 9 illustrates the agreement between the approximated and the exacts results. It is valid only for the high temperature region. At low $T$, the main contribution to the partition function comes from the ground state. The lowest energy is the one of the configuration where all states in the lower level are occupied while the ones in the upper level are empty. This corresponds to the case $(2 \tau)=2 \Omega, \nu_{1}=\nu_{2}=0$. In the approximation, the partition function reduces to one term, given by the integral (26) with $J=\Omega$, and the energy goes to $-2 V_{1}^{2} \Omega^{4} / \omega_{b}$ as $T$ goes to zero. This value, is of the order of $-13 \mathrm{GeV}$ if one uses the already given parameters $\left(V_{1}=0.04 \mathrm{GeV}, \Omega=9\right.$, and $\omega_{b}$ $=1.6 \mathrm{GeV})$. The exact calculation yields $-2 \mathrm{GeV}$. This deviation is caused by the approximation (18).

Note that, in a more realistic context, the transition to the QGP is believed to take place around $T=0.165 \mathrm{GeV}$ [14]. For these temperatures our approximate results are acceptable. Also, for these temperatures there is still a sizable difference between the results obtained with interactions and without interactions. It shows also that the interactions cannot be neglected in the high temperature regime.

Finally, we like to discuss the dependence of $T$ on the chemical potential $\mu$, when the pressure of the system is equal to the bag pressure $B=0.145 \mathrm{GeV}$ [24]. To find such a dependence one has to introduce a volume. We consider an elementary volume given by the size of a hadron $\left(\approx 1 \mathrm{fm}^{3}\right)$, as done in Ref. [13]. The pressure is then given by the ratio of the internal energy and this volume. The result is depicted in Fig. 11. Without interactions $\left(V_{1}=0\right)$ we reproduce the results of Ref. [24]. By turning on the interaction $\left(V_{1}=0.04 \mathrm{GeV}\right)$, the chemical potential increases. This effect shows that the correlated vacuum state is dominated by gluon pairs and it also has contributions from quarkantiquark pairs.
We have shown here that the exact result can be approximated with the procedure exposed. It has the possibility to be extended to arbitrary Hamiltonians with interactions, which in addition do not have a simpler structure due to symmetries.

\section{CONCLUSIONS}

In this work we have introduced the essentials of a toy model for QCD. The model consists of two levels with energies $\pm \omega_{f}$, which describe the fermion degrees of freedom, and gluons are introduced via a level of positive energy which can be filled by gluon pairs with spin zero. The gluon pairs are treated as bosons. Two different Hamiltonians are discussed. The first one $\left(\boldsymbol{H}_{I}\right)$ commutes with the symmetry operator $\boldsymbol{P}$, a property which allows us to calculate the energy spectrum easily. However, this Hamiltonian contains only a certain type of interaction terms which do not include ground-state correlations. The second Hamiltonian $\left(\boldsymbol{H}_{I I}\right)$ does contain terms which produce ground-state correlations. Due to the symmetry of the vertices, respect to the exchange of fermion and boson lines, all terms entering in $\boldsymbol{H}_{I I}$ have the same interaction strength. We have shown that the corresponding spectrum exhibits a quantum phase transition, depending on the interaction. For small values of the interaction $V_{1}$, fermion pairs and gluon pairs equally populate the ground state, for intermediate values of $V_{1}$ the physical vacuum is described by quark-antiquark pairs while for larger values of $V_{1}$ gluon pairs dominate. In the gluon dominated phase the spectrum has a degeneracy of the ground state, given by one positive- and one negative-parity state. This property gives some hope that a more general version of the model, with open flavor and spin channels, may show a Goldstone boson [in the flavor octet $(1,1) \mathrm{SU}(3)$ notation], if the strength of the corresponding interaction is large enough. The appearance of the gluon and quark-antiquark condensate and of the Goldstone boson may be easily described by using coherent states, as we have shown for the present version of the model. The same approach may be useful for the case of more general models of nonperturbative QCD. Important to note is that the states at low energy will consist of a not definite number of quarks, antiquarks, and gluons. The nonconservation of the particle number will be essential in order to remove the multiplicity of states encountered in models with no particle mixing interaction. There, a given state can be constructed in various manners by different realizations. The mesons (and in general hadrons) will contain more than just the valence quarks and antiquarks.

Baryons were also considered in the model [see Eq. (8)] and due to Pauli-blocking effect the effective degeneracy $\Omega$ decreases. Also, the transition point to a condensate of pairs of quarks-antiquarks and gluons is shifted to larger values of the interaction strength. There is a regime where mesons are already in a condensate phase while the baryons can still be treated as three quark systems.

We have investigated finite temperature effects, by constructing the partition function of the model, both exactly and approximately. A method was chosen which allows a control over the approximations made and it can be extended 
to more realistic systems where, however, an exact solution is impossible or very difficult to obtain. We have shown that the use of coherent states makes it possible to introduce approximations in a controlled way. The results, for the internal energy, heat capacity, and the equation of state of the system at the bag pressure are in agreement with previous calculations. The model gives us the possibility to describe in a continuous manner the transition from low energy to the QGP, unique up to now. The model predicts a first order transition to the QGP.

To summarize, the model is able to describe characteristic features of QCD at low and at high temperature. This gives some hope that, in a more general version of it with open flavor and color [17], it may describe the hadron spectrum at low energy and the transition to the QGP as well.

\section{ACKNOWLEDGMENTS}

We acknowledge financial support through the CONACyT-CONICET agreement under the project name Algebraic Methods in Nuclear and Subnuclear Physics and from CONACyT Project No. 32729-E. S.J. acknowledges financial support from the Deutscher Akademischer Austauschdienst (DAAD) and SRE, S.L. acknowledges financial support from DGEP-UNAM.

\section{APPENDIX}

We want to calculate the expectation values of the operators $\boldsymbol{S}_{0},\left(\boldsymbol{S}_{+}^{2}+\boldsymbol{S}_{-}^{2}\right)$, and $\boldsymbol{S}_{+} \boldsymbol{S}_{-}$using coherent states. We adopted, as a suitable representation, the normalized coherent states [20],

$$
\left|z_{f}\right\rangle=\frac{1}{\left(1+\left|z_{f}\right|\right)^{J}} e^{z_{f} \boldsymbol{S}_{+}}|J,-J\rangle
$$

where $|J,-J\rangle$ is the eigenstate of the pseudospin algebra with the absolute value of the spin $J$ and its projection $M$ $=-J$.

For the operator $\boldsymbol{S}_{0}$ we have

$$
\left\langle z_{f}\left|\boldsymbol{S}_{0}\right| z_{f}\right\rangle=\frac{1}{\left(1+\left|z_{f}\right|^{2}\right)^{2 J}}\left\langle J,-J\left|\left(e^{z_{f}^{\star}} \boldsymbol{S}_{-} \boldsymbol{S}_{0} e^{z} \boldsymbol{S}_{+}\right)\right| J,-J\right\rangle .
$$

Now, since $\left[\boldsymbol{S}_{-}, \boldsymbol{S}_{0}\right]=\boldsymbol{S}_{-}$and

$$
e^{A} \boldsymbol{O} e^{-\boldsymbol{A}}=\boldsymbol{O}+\frac{1}{1 !}[\boldsymbol{A}, \boldsymbol{O}]+\frac{1}{2 !}[\boldsymbol{A},[\boldsymbol{A}, \boldsymbol{O}]]+\cdots
$$

we arrive at

$$
e^{z_{f}^{\star} \boldsymbol{S}_{-}} \boldsymbol{S}_{0} e^{z_{f} \boldsymbol{S}_{+}}=\left(\boldsymbol{S}_{0}+z_{f}^{\star} \boldsymbol{S}_{-}\right) e^{z_{f}^{\star} \boldsymbol{S}_{-}} e^{z_{f} \boldsymbol{S}_{+}}
$$

This yields

$$
\begin{aligned}
\left\langle z_{f}\left|\boldsymbol{S}_{0}\right| z_{f}\right\rangle= & \frac{1}{\left(1+\left|z_{f}\right|^{2}\right)^{2 J}}\langle J,-J|\left(\boldsymbol{S}_{0}+z_{f}^{\star} \boldsymbol{S}_{-}\right) \\
& \times e^{z_{f}^{\star} \boldsymbol{S}_{-} e^{z} \boldsymbol{S}_{+}}|J,-J\rangle \\
= & \frac{1}{\left(1+\left|z_{f}\right|^{2}\right)^{2 J}}\left(-J+z_{f}^{\star} \frac{\partial}{\partial z_{f}}\right) \\
& \times\left\langle J,-J\left|e^{z_{f}^{\star}} \boldsymbol{S}_{-} e^{z_{f} \boldsymbol{S}_{+}}\right| J,-J\right\rangle .
\end{aligned}
$$

To calculate the normalization, $\left\langle J,-J\left|e^{z_{f}^{\star}} S_{-} e^{z_{f} S_{+}}\right| J\right.$, $-J\rangle$, we write [20]

$$
\left(1+\left|z_{f}\right|\right)^{J}\left|z_{f}\right\rangle=e^{z} S_{+}|J,-J\rangle=\sum_{n=0}^{2 J} \frac{\left|z_{f}\right|^{n}}{\sqrt{n !}} \sqrt{\frac{(2 J) !}{(2 J-n) !}}|J, n\rangle
$$

leading to $\left\langle J,-J\left|e^{z_{f}^{\star} S_{-}} e^{z_{f} \boldsymbol{S}_{+}}\right| J,-J\right\rangle=\left(1+\left|z_{f}\right|^{2}\right)^{2 J}$.

With this result, the expectation value of $\boldsymbol{S}_{0}$ reads

$$
\begin{aligned}
\left\langle z_{f}\left|\boldsymbol{S}_{0}\right| z_{f}\right\rangle & =\frac{1}{\left(1+\left|z_{f}\right|^{2}\right)^{2 J}}\left(-J+z_{f}^{\star} \frac{\partial}{\partial z_{f}^{\star}}\right)\left(1+\left|z_{f}\right|^{2}\right)^{2 J} \\
& =-\frac{J\left(1-\left|z_{f}\right|^{2}\right)}{1+\left|z_{f}\right|^{2}} .
\end{aligned}
$$

Next we are going to calculate the expectation value of the operator $\boldsymbol{S}_{+}^{2}$,

$$
\begin{aligned}
\left\langle z_{f}\left|\boldsymbol{S}_{+}^{2}\right| z_{f}\right\rangle & =\frac{1}{\left(1+\left|z_{f}\right|^{2}\right)^{2 J}}\left\langle J,-J\left|\left(e^{z_{f}^{\star}} \boldsymbol{S}_{-} \boldsymbol{S}_{+}^{2} e^{z_{f} \boldsymbol{S}_{+}}\right)\right| J,-J\right\rangle \\
& =\frac{1}{\left(1+\left|z_{f}\right|^{2}\right)^{2 J}} \frac{\partial^{2}}{\partial z_{f}^{2}}\left\langle J,-J\left|\left(e^{z_{f}^{\star} \boldsymbol{S}_{-}} e^{z_{f} \boldsymbol{S}_{+}}\right)\right| J,-J\right\rangle \\
& =\frac{1}{\left(1+\left|z_{f}\right|^{2}\right)^{2 J}} \frac{\partial^{2}}{\partial z_{f}^{2}}\left(1+\left|z_{f}\right|^{2}\right)^{2 J} \\
& =\frac{2 J(2 J-1)\left(z_{f}^{\star}\right)^{2}}{\left(1+\left|z_{f}\right|^{2}\right)^{2}} .
\end{aligned}
$$

Therefore

$$
\left\langle z_{f}\left|\left(\boldsymbol{S}_{+}^{2}+\boldsymbol{S}_{-}^{2}\right)\right| z_{f}\right\rangle=\frac{2 J(2 J-1)\left[\left(z_{f}^{\star}\right)^{2}+z_{f}^{2}\right]}{\left(1+\left|z_{f}\right|^{2}\right)^{2}} .
$$

To calculate the expectation value of $\boldsymbol{S}_{+} \boldsymbol{S}_{-}$we use the identity $\boldsymbol{S}_{+} \boldsymbol{S}_{-}=\boldsymbol{S}^{2}-\boldsymbol{S}_{0}^{2}+\boldsymbol{S}_{0}$, and from the above obtained results we find 


$$
\begin{aligned}
\left\langle z_{f}\left|\boldsymbol{S}_{0}^{2}\right| z_{f}\right\rangle & =\frac{1}{\left(1+\left|z_{f}\right|^{2}\right)^{2 J}}\left(-J+z_{f}^{\star} \frac{\partial}{\partial z_{f}^{\star}}\right)^{2}\left(1+\left|z_{f}\right|^{2}\right)^{2 J} \\
& =J^{2}-\frac{4 J^{2}\left|z_{f}\right|^{2}}{1+\left|z_{f}\right|^{2}}+\frac{2 J\left|z_{f}\right|^{2}}{1+\left|z_{f}\right|^{2}}+\frac{2 J(2 J-1)\left|z_{f}\right|^{4}}{\left(1+\left|z_{f}\right|^{2}\right)^{2}} \\
& =J^{2}-\frac{2 J(2 J-1)\left|z_{f}\right|^{2}}{\left(1+\left|z_{f}\right|^{2}\right)^{2}}
\end{aligned}
$$

and

$$
\begin{aligned}
\left\langle z_{f}\left|\boldsymbol{S}_{+} \boldsymbol{S}_{-}\right| z_{f}\right\rangle= & \left\langle z_{f}\left|\left(\boldsymbol{S}^{2}-\boldsymbol{S}_{0}^{2}+\boldsymbol{S}_{0}\right)\right| z_{f}\right\rangle \\
= & J(J+1)-\left(J^{2}-\frac{2 J(2 J-1)\left|z_{f}\right|^{2}}{\left(1+\left|z_{f}\right|^{2}\right)^{2}}\right) \\
& -\frac{J\left(1-\left|z_{f}\right|^{2}\right)}{1+\left|z_{f}\right|^{2}} \\
= & \frac{2 J\left|z_{f}\right|^{2}\left(2 J+\left|z_{f}\right|^{2}\right)}{\left(1+\left|z_{f}\right|^{2}\right)^{2}} .
\end{aligned}
$$

Finally, in the limit of large $J$ expressions like $(2 J-k)$ are approximated by $2 J$.
[1] H. J. Lipkin, N. Meschkov, and S. Glick, Nucl. Phys. A62, 118 (1965).

[2] D. Schütte and J. Da Providencia, Nucl. Phys. A282, 518 (1977)

[3] O. Civitarese, P. O. Hess, J. G. Hirsch, and M. Reboiro, Phys. Rev. C 61, 064303 (2000).

[4] J. Dobes and S. Pittel, Phys. Rev. C 57, 688 (1998).

[5] J. G. Hirsch, P. O. Hess, and O. Civitarese, Phys. Lett. B 390, 36 (1997); O. Civitarese, P. O. Hess, and J. G. Hirsch, ibid. 412, 1 (1997); J. G. Hirsch, P. O. Hess, and O. Civitarese, Phys. Rev. C 56, 199 (1997).

[6] A. Klein and E. R. Marshalek, Rev. Mod. Phys. 63, 375 (1991).

[7] H. J. Rothe, Lattice Gauge Theories: An Introduction (World Scientific, Singapore, 1992).

[8] G. S. Bali, K. Schilling, A. Hulsebos, A. C. Irving, C. Michael, and P. W. Stephenson, Phys. Rev. B 309, 378 (1993); M. Peardon, Nucl. Phys. B (Proc. Suppl.) 63, 22 (1998).

[9] P. O. Hess, S. Lerma, J. C. López, C. R. Stephens, and A. Weber, Eur. Phys. J. C 9, 121 (1999).

[10] A. P. Szczepaniak, E. S. Swanson, C.-R. Jia, and S. R. Cotanch, Phys. Rev. Lett. 76, 2011 (1996); E. S. Swanson and A. P. Szczepaniak, Phys. Rev. D 56, 5692 (1997); P. Page, E. S. Swanson, and A. P. Szczepaniak, ibid. 59, 034016 (1999); E. S. Swanson and A. P. Szczepaniak, ibid. 59, 014035 (1999).

[11] U. Löring, B. Ch. Metsch, and H. R. Petry, Eur. Phys. J. A 10, 309 (2001); 10, 395 (2001); 10, 447 (2001).
[12] H. Y. Cheng, Int. J. Mod. Phys. A 11, 5109 (1996).

[13] B. Müller, The Physics of the Quark-Gluon Plasma, Lecture Notes in Physics Vol. 225 (Springer, Heidelberg, 1985).

[14] S. A. Bass, nucl-th/0202010 v2, Pramana (to be published), and references therein.

[15] K. Langfeld, J. Reimhardt, and J. Gattner, Nucl. Phys. B621, 131 (2002).

[16] J. P. Draayer and Y. Akiyama, J. Math. Phys. 14, 1904 (1973).

[17] S. Lerma, S. Jesgarz, P. O. Hess, O. Civitarese, and M. Reboiro (unpublished).

[18] J. P. Elliott and P. G. Dawber, Symmetry in Physics (Oxford University Press, New York, 1974), Vols. 1 and 2.

[19] P. Ring and P. Schuck, The Nuclear Many-Body Problem (Springer-Verlag, Heidelberg, 1980).

[20] K. T. Hecht, The Vector Coherent State Method and Its Applications to Physical Problems of Higher Symmetries, Lecture Notes in Physics Vol. 290 (Springer-Verlag, Heidelberg, 1987).

[21] O. Civitarese, M. Reboiro, S. Jesgarz, and P. O. Hess, Phys. Rev. C 64, 054317 (2001).

[22] A. Kuriyama, J. da Providencia, Y. Tsue, and M. Yamamura, Prog. Theor. Phys. 94, 1039 (1995); A. Kuriyama, J. da Providencia, C. da Providencia, Y. Tsue, and M. Yamamura, ibid. 95, 339 (1996).

[23] E. Merzbacher, Quantum Mechanics (Wiley, New York, 1998), p. $38 f$.

[24] W. Greiner, L. Neise, and H. Stöcker, Thermodynamics and Statistical Mechanics (Springer-Verlag, Heidelberg, 1994). 